\title{
Alternative Estimation Method for a Three-Stage Cluster Sampling in Finite Population
}

\author{
L. A. Nafiu ${ }^{1, *}$, I. O. Oshungade ${ }^{2}$, A. A. Ade wara \\ ${ }^{1}$ Department of Mathematics and Statistics, Federal University of Technology, Minna, +234, Nigeria \\ ${ }^{2}$ Department of Statistics, University of Ilorin, Ilorin, +234 , Nigeria
}

\begin{abstract}
This research investigates the use of a three-stage cluster sampling design in estimating population total. We focus on a special design where certain number of visits is being considered for estimating the population size and a weighted factor of / is introduced. In particular, attempt was made at deriving new method for a three-stage sampling design. In this study, we compared the newly proposed estimator with some of the existing estimators in a three-stage sampling design. Eight (8) data sets were used to justify this paper. The first four (4) data sets were obtained fro m[1],[2],[3] and[4] respectively while the second four (4) data sets represent the number of diabetic patients in Niger state, Nigeria for the years 2005, 2006, 2007 and 2008 respectively. The computation was done with software developed in Microsoft Visual C++ programming language. All the estimates obtained show that our newly proposed three-stage cluster sampling design estimator performs better.
\end{abstract}

\section{Keywo rds Sampling, Three-stage, Cluster Design, Estimator, Bias and Variance}

\section{Introduction}

In a census, each unit (such as person, household or local government area) is enumerated, whereas in a sample survey, only a sample of units is enumerated and information provided by the sample is used to make estimates relating to all units[5] and[6]. In designing a study, it can be advantageous to sample units in more than one-stage. The criteria for selecting a unit at a given stage typically depend on attributes observed in the previous stages[7]. Multistage sampling is where the researcher divides the population into clusters, samples the clusters, and then resample, repeating the process until the ultimate sampling units are selected at the last of the hierarchical levels[8]. If, after selecting a sample of primary units, a sample of secondary units is selected fro meach of the selected primary units, the design is referred to as two-stage sampling. If in turn a sample of tertiary units is selected from each selected secondary unit, the design is three-stage sampling[9].

The aim of this paper is to model a new estimator for three-stage cluster samp ling scheme which is to be co mpared with the other existing seven conventional estimators.

\section{Methodology}

* Corresponding author:

lanconserv@yahoo.com (L. A. Nafiu)

Published online at http://journal.sapub.org/ajms

Copyright (C) 2012 Scientific \& Academic Publishing. All Rights Reserved
Sub sampling has a great variety of applications [3] and the reason for multistage sampling is administrative convenienc $\mathrm{e}[10]$. The process of sub sampling can be carried to a third stage by sampling the subunits instead of enumerating them completely[11]. Co mparing mu lt is tage cluster sampling with simple random sampling, it was observes that multistage cluster sampling is better in terms of efficiency[12]. Multistage sampling makes fieldwork and supervision relatively easy[4]. Multistage sampling is more efficient than single stage cluster sampling[13] and references had been made to the use of three or more stages sampling[9].

Let $N$ denote the number of primary units in the population and $n$ the number of primary units in the sample. Let $M_{i}$ be the number of secondary units in the primary unit. The total number of secondary units in the population is

$$
M=\sum_{i=1}^{N} M_{i}
$$

Let $y_{i j}$ denote the value of the variable of interest of the $j^{\text {th }}$ secondary unit in the $i^{\text {th }}$ primary unit. The total of the $y$-values in the ith primary unit is

$$
Y_{i}=\sum_{j=1}^{M_{i}} y_{i j}
$$

Accordingly, the population total for over-all sample in a two-stage is given as

$$
Y=\sum_{i=1}^{N} \sum_{j=1}^{M_{i}} y_{i j}
$$

For a three-stage sampling, the population contains $\mathrm{N}$ 
first-stage units, each with $\mathrm{M}$ second-stage units, each of which has $\mathrm{K}$ third-stage units. The corresponding numbers for the sample are $\mathrm{n}, \mathrm{m}$ and $\mathrm{k}$ respectively. Let $y_{i j u}$ be the value obtained for the $\mathrm{u}^{\text {th }}$ third-stage units in the $\mathrm{j}^{\text {th }}$ second-stage units drawn from the ith primary units. The relevant population total for over-all sample in a three-stage is given as follows:

$$
Y=\sum_{i=1}^{N} \sum_{j=1}^{M} \sum_{u=1}^{K} y_{i j u}
$$

For any estimation $\Theta_{h}$ in the $\mathrm{h}^{\text {th }}$ cell based on completely arbitrary probabilities of selection, the total variance is then the sum of the variances for all strata. The symbol $\mathrm{E}$ is used for the operator of expectation, $\mathrm{V}$ for the variance, and $V$ for the unbiased estimate of V. We may then write

$$
V\left(\Theta_{h}\right)=V_{1}\left(\underset{>1}{E}\left(\Theta_{h}\right)\right)+\underset{1}{E}\left(\underset{>1}{V}\left(\Theta_{h}\right)\right)
$$

The expression (5) may be written into three components as:

$$
V\left(\Theta_{h}\right)=\underset{1}{V}\left(\underset{2}{E}\left(\underset{2}{E}\left(\Theta_{h}\right)\right)\right)+\underset{1}{E}\left(\underset{2}{V}\left(\underset{2}{E}\left(\Theta_{h}\right)\right)\right)+\underset{1}{E}\left(\underset{2}{E}\left(\underset{V}{(V}\left(\Theta_{h}\right)\right)\right)
$$

where " $>1$ " is the symbol to represent all stages of sampling after the first[3].

\section{Proposed Three-Stage Cluster Sampling Design}

To estimate the population size at different hospitals using three-stage sampling, the unbiased estimator of population total can be derived as follows. In a three-stage sampling without replacement design supported by[3],[4] and[14]; a sample of primary units is selected, then a sample of secondary units is chosen from each of the selected primary units and finally, a sample of tertiary units is chosen from each selected secondary unit. For instance, the state consists of $N$ number of local government areas out of which a simple random sampling of $\mathrm{n}$ number of local government areas is selected. Each local government area consists of $M_{i}$ number of cities out of which a simple random sampling without replacement of $m_{i}$ number of cities is selected. Finally, from the selected sample of city containing $K_{i j}$ number of hospitals, $k_{i j}$ number of hospitals is selected at random without replacement and the number of diabetic patients in this hospital is collected.

Then;

$$
y=\sum_{i=1}^{n} \sum_{j=1}^{m_{i}} y_{i j}
$$

Again, let $n$ be the number of primary units (local government areas) sampled without replacement, $m_{i}$ be the number of secondary units (cities) selected without replacement from the ith sampled primary unit (local government area) and $k_{i j}$ be the number of tertiary units (hospitals) selected from the $j$ th secondary unit (city) in the ith primary unit (local government area). An unbiased estimator of the population total at $j$ th secondary unit in the ith primary unit in the sample is:

$$
\begin{gathered}
\hat{y}_{i j}=\frac{1}{\gamma_{i j}} \sum_{l=1}^{k_{i j}} \frac{N_{i}}{n_{i}^{2}} y_{i j l} \\
=\frac{K_{i j}}{k_{i j}} \sum_{l=1}^{k_{i j}} \frac{N_{i}}{n_{i}^{2}} y_{i j l}
\end{gathered}
$$

where $\gamma_{i j}=\frac{k_{i j}}{K_{i j}}$ is the known sampling fraction for tertiary units in the $j t h$ secondary unit of the $i t h$ primary unit.

Also, let $y_{i j l}$ denote the number of individuals (tertiary units) in the sample from the $j t h$ secondary unit of the $i t h$ primary unit who engage in the treatment of diabetes. An unbiased estimator of the population total in the ith primary unit in the sample is:

$$
\hat{y_{i}}=\frac{M_{i}}{m_{i}} \sum_{j=1}^{m_{i}} \hat{y}_{i j}
$$

Finally, an unbiased estimator of the population total of the diabetic patients undergoing treatment in all the hospitals at the $j t h$ secondary unit (city) in the ith primary unit (local government area) is:

$$
\begin{gathered}
\widehat{Y}_{3 N P E}=\frac{N}{n} \sum_{i=1}^{n} \hat{y}_{i} \\
=\frac{N}{n} \sum_{i=1}^{n}\left\{\frac{M_{i}}{m_{i}} \sum_{j=1}^{m_{i}}\left(\frac{K_{i j}}{k_{i j}} \sum_{l=1}^{k_{i j}} \frac{N_{i}}{n_{i}^{2}} y_{i j l}\right)\right\}
\end{gathered}
$$

\section{Theorems}

\subsection{Theorem 1: $\widehat{P}_{3 N P E}$ is Unbiased for the Population total $\mathbf{Y}$}

\section{Proof:}

We know that expectation of $\hat{y}_{i j}$ given by equation (8) conditional on samples $s_{1}$ and $s_{2}$ of primary units and secondary units respectively equals $y_{i j}$ of engaging in the variable of interest in each primary unit and each secondary unit [15]. That is;

$$
E\left(\hat{y}_{i j} \mid s_{1}, s_{2}\right)=y_{i j}
$$

Also, the expectation of $\hat{y}_{i}$ given by equation (9) conditional on sample $s_{1}$ of primary units equals $y_{i}$ of engaging in the variable of interest in each primary unit.

That is;

$$
E\left(\hat{y}_{i} \mid s_{1}\right)=y_{i}
$$

To obtain the expected value of $\hat{Y}_{3 N P E}$ given by equation (10) over all possible samples of primary units.

Then, the expectation of $\hat{Y}_{3 N P E}$ is :

$$
E\left(\widehat{Y}_{3 N P E}\right)=E_{1}\left[E_{2}\left\{E_{3}\left(\widehat{Y}_{3 N P E} \mid s_{1}, s_{2}\right) \mid s_{1}\right\}\right]
$$

where $s_{1}$ and $s_{2}$ denote the samples of primary units and secondary units respectively.

$$
\begin{gathered}
E\left(\hat{Y}_{3 N P E}\right)=E\left[E\left\{E\left(\frac{N}{n} \sum_{i=1}^{n} \hat{y}_{i j} \mid s_{1}, s_{2}\right) \mid s_{1}\right\}\right] \\
=E\left[E\left\{\frac{N}{n} \sum_{i=1}^{n} \hat{y}_{i} \mid s_{1}\right\}\right] \\
=E\left\{\frac{N}{n} \sum_{i=1}^{n} y_{i}\right\}
\end{gathered}
$$




$$
\begin{aligned}
& =\frac{N}{n} \frac{n}{N} \sum_{i=1}^{N} y_{i} \\
& =Y
\end{aligned}
$$

This implies that the proposed estimator $\hat{Y}_{3 N P E}$ is unbiased.

Hence, the variance of the newly proposed estimator $\widehat{Y}_{3 N P E}$ of the population total is derived as follows:

In line with[3] and[14], we use

$$
\begin{aligned}
V\left(\hat{Y}_{3 N P E}\right)=V\{E & \left.\left(\hat{Y}_{3 N P E} \mid s_{1}, s_{2}\right) \mid s_{1}\right\} \\
& +E\left\{V\left[\left(\hat{Y}_{3 N P E} \mid s_{1}, s_{2}\right) \mid s_{1}\right]\right\}=
\end{aligned}
$$

$V\left\{E\left(\hat{Y}_{3 N P E}\right)\right\}+E\left\{V\left[E\left(\hat{Y}_{3 N P E}\right)\right]\right\}+E\left\{E\left[V\left(\hat{Y}_{3 N P E}\right)\right]\right\}$

Because of the simple random sampling of primary units and secondary units without replacement at the first stage and second stage respectively, the first termto the right of the equality in equation (15) is:

$$
V\left\{E\left(\hat{Y}_{3 N P E} \mid s_{1}, s_{2}\right) \mid s_{1}\right\}=V\left\{\frac{N}{n} \sum_{i=1}^{n} y_{i}\right\}=\frac{N(N-n)}{n} \sigma_{1}^{2}
$$

The second term to the right of the equality in equation (15) is:

$$
\begin{gathered}
V\left\{\left(\hat{Y}_{3 N P E} \mid s_{1}, s_{2}\right) \mid s_{1}\right\}=V\left\{\frac{N}{n} \sum_{i=1}^{n} \hat{y}_{i} \mid s_{1}\right\} \\
=\left(\frac{N}{n}\right) \sum_{i=1}^{n} V\left(\hat{y}_{i} \mid s_{1}\right)+\left(\frac{N}{n}\right)^{2} \sum_{i=1}^{n} V\left(\hat{Y}_{3 N P E}\right) \\
=\left(\frac{N}{n}\right) \sum_{i=1}^{n} \sigma_{i j}^{2}+\left(\frac{N}{n}\right)^{2} \sum_{i=1}^{n} \sigma_{i}^{2} \\
E\left[V\left\{\left(\hat{Y}_{3 N P E} \mid s_{1}, s_{2}\right) \mid s_{1}\right\}\right] \\
=\frac{N}{n} \sum_{i=1}^{N} \frac{M_{i}}{m_{i}} \sum_{j=1}^{M_{i}} K_{i j}\left(K_{i j}-k_{i j}\right) \frac{\sigma_{i j}^{2}}{k_{i j}} \\
+\left(\frac{N}{n}\right)^{2} \frac{n}{N} \sum_{i=1}^{N} M_{i}\left(M_{i}-m_{i}\right) \frac{\sigma_{i}^{2}}{m_{i}} \\
=\frac{N}{n} \sum_{i=1}^{N} M_{i}\left(M_{i}-m_{i}\right) \frac{\sigma_{i}^{2}}{m_{i}}+\frac{N}{n} \sum_{i=1}^{N} \frac{M_{i}}{m_{i}} \sum_{j=1}^{M_{i}} K_{i j}\left(K_{i j}-\right.
\end{gathered}
$$

Equations (16) and (17) give;

$$
\begin{gathered}
V\left(\hat{Y}_{3 N P E}\right)=N(N-n) \frac{\sigma_{1}^{2}}{n}+\frac{N}{n} \sum_{i=1}^{N} M_{i}\left(M_{i}-m_{i}\right) \frac{\sigma_{i}^{2}}{m_{i}}+ \\
N n i=1 \text { NMimij=1MiKijKij-kijoij2kij }
\end{gathered}
$$

where

$$
\begin{gathered}
\sigma_{1}^{2}=\frac{\sum_{i=1}^{N}\left(Y_{i}-\bar{Y}\right)^{2}}{N-1_{Y_{i}}} \\
\sigma_{i}^{2}=\frac{\sum_{j=1}^{M_{i}\left(Y_{i j}-\frac{M_{i}}{M_{i}}\right)^{2}}}{M_{i}-1} \\
\sigma_{i j}^{2}=\frac{K_{i j}^{2}}{k_{i j}^{2}} \sum_{l=1}^{k_{i j}}\left(\frac{N_{i}^{2}}{n_{i}^{4}}-\frac{N_{i}}{n_{i}^{2}}\right)\left(y_{i j l}-\bar{y}_{i j}\right)^{2}
\end{gathered}
$$

We note that the first term to the right of the equality in equation (18) is the variance that would be obtained if every tertiary unit in a selected secondary unit and every secondary unit in a selected primary unit was observed, that is, if $y_{i}$ 's were known for $i=1,2, \cdots, n$. The second term contains variance that would be obtained if every tertiary unit in a selected secondary unit was observed, that is, if $y_{i j}$ 's were known for $i=1,2, \cdots, n$ and $j=1,2, \cdots, m_{i}$. The third term contains variance due to estimating the $y_{i j}$ 's from a subsample of tertiary units within the selected secondary units. An unbiased estimator of the variance of $\hat{Y}_{3 N P E}$ given in equation (18) is obtained by replacing the population variances with the sample variances as follows:

$$
\begin{array}{r}
\widehat{V}\left(\hat{Y}_{3 N P E}\right)=N(N-n) \frac{s_{1}^{2}}{n}+\frac{N}{n} \sum_{i=1}^{n} M_{i}\left(M_{i}-m_{i}\right) \frac{s_{i}^{2}}{m_{i}}+ \\
N n i=1 \text { nMimij=1miKijKij-kijsij2kij }(22)
\end{array}
$$

where

$$
\begin{gathered}
s_{1}^{2}=\frac{\sum_{i=1}^{n}\left(y_{i}-\frac{\widehat{Y}_{3 N P E}}{n}\right)^{2}}{n-1} \\
S_{i}^{2}=\frac{\sum_{j=1}^{m_{i}}\left(y_{i j}-\frac{y_{i}}{m_{i}}\right)^{2}}{m_{i}-1} \\
s_{i j}^{2}=\frac{K_{i j}^{2}}{k_{i j}^{2}} \sum_{l=1}^{k_{i j}}\left(\frac{N_{i}^{2}}{n_{i}^{4}}-\frac{N_{i}}{n_{i}^{2}}\right)\left(y_{i j l}-\bar{y}_{i j}\right)^{2}
\end{gathered}
$$

\subsection{Theorem 2: $\widehat{V}\left(\widehat{Y}_{3 N P E}\right)$ is Unbiased for $V\left(\widehat{Y}_{3 N P E}\right)$}

Proof:

We note that

$$
s_{1}^{2}=\frac{1}{n-1}\left(\sum_{i=1}^{n} y_{i}^{2}-\frac{n \Upsilon_{3 N P E}^{2}}{N^{2}}\right)
$$

Next, we note that

$$
\begin{gathered}
E\left(\sum_{i=1}^{n} y_{i}^{2}\right)=E\left\{E\left(\sum_{i=1}^{n} y_{i}^{2} \mid s_{1}\right)\right\} \\
=E\left(\sum_{i=1}^{n}\left[V\left(y_{i} \mid s_{1}\right)+\left\{E\left(y_{i} \mid s_{1}\right)\right\}^{2}\right]\right) \\
=E\left(\sum_{i=1}^{n} \sigma_{i}^{2}+\sum_{i=1}^{n} Y_{i}^{2}\right) \\
=E\left(\sum_{i=1}^{N} z_{i} \sigma_{i}^{2}+\sum_{i=1}^{N} z_{i} Y_{i}^{2}\right) \\
=\frac{n}{N}\left(\sum_{i=1}^{N} \sigma_{i}^{2}+\sum_{i=1}^{N} Y_{i}^{2}\right)
\end{gathered}
$$

In addition;

$$
\begin{aligned}
E\left(\hat{Y}_{3 N P E}^{2}\right) & =V\left(\hat{Y}_{3 N P E}\right)+\left\{E\left(\hat{Y}_{3 N P E}\right)\right\}^{2} \\
& =\frac{N(N-n)}{n} \sigma_{1}^{2}+\frac{N}{n} \sum_{i=1}^{N} \sigma_{i}^{2}+Y^{2}
\end{aligned}
$$

Combining equations (27) and (28), we have:

$$
E\left(s_{1}^{2}\right)=
$$

$$
\frac{n}{N(n-1)}\left(\sum_{i=1}^{N} \sigma_{i}^{2}+\sum_{i=1}^{N} Y_{i}^{2}\right)-\frac{n}{N^{2}(n-1)}\left(\frac{N(N-n)}{n} \sigma_{1}^{2}+\right.
$$

$$
N n i=1 N \sigma i 2+Y 2)
$$

$=\frac{1}{N} \sum_{i=1}^{N} \sigma_{i}^{2}+\frac{(N-1)_{n}}{(n-1) N}\left\{\frac{1}{N-1}\left(\sum_{i=1}^{N} Y_{i}^{2}-\frac{Y^{2}}{N}\right)\right\}+\frac{(N-n)}{N(n-1)} \sigma_{1}^{2}(29)$

Using the fact that:

$$
\sigma_{1}^{2}=\frac{1}{N-1}\left(\sum_{i=1}^{N} Y_{i}^{2}-\frac{Y^{2}}{N}\right)
$$

Equation (29) becomes;

$$
\begin{aligned}
E\left(s_{1}^{2}\right)= & \frac{1}{N} \sum_{i=1}^{N} \sigma_{i}^{2}+\frac{(N-1) n}{(n-1) N} \sigma_{1}^{2}-\frac{(N-n)}{N(n-1)} \sigma_{1}^{2} \\
= & \frac{1}{N} \sum_{i=1}^{N} \sigma_{i}^{2}+\frac{N(N-n)}{n} \sigma_{1}^{2}
\end{aligned}
$$

Next, we note that; 


$$
\begin{gathered}
E\left(s_{i}^{2}\right)=E\left\{E\left(\sum_{i=1}^{n} s_{i}^{2}\right)\right\} \\
=E\left\{E\left(\sum_{i=1}^{n} s_{i}^{2}\right)\right\} \\
=E\left\{E\left(\sum_{i=1}^{n} s_{i}^{2} \mid s_{1}\right)\right\} \\
=E\left\{\sum_{i=1}^{n} \frac{M_{i}^{2}}{m_{i}^{2}} \sigma_{i}^{2}\right) \\
=E\left\{\sum_{i=1}^{N} z_{i} \frac{M_{i}^{2}}{m_{i}^{2}} \sigma_{i}^{2}\right) \\
=\frac{n}{N}\left\{\frac{N^{2}}{n^{2}} \sum_{i=1}^{N} M_{i}\left(M_{i}-m_{i}\right) \frac{\sigma_{i}^{2}}{m_{i}}-\frac{1}{n} \sum_{i=1}^{N} M_{i}\left(M_{i}-m_{i}\right) \frac{\sigma_{i}^{2}}{m_{i}}\right\} \\
=\frac{N}{n} \sum_{i=1}^{N} M_{i}\left(M_{i}-m_{i}\right) \frac{\sigma_{i}^{2}}{m_{i}}-\frac{1}{N} \sum_{i=1}^{N} M_{i}\left(M_{i}-m_{i}\right) \frac{\sigma_{i}^{2}}{m_{i}}
\end{gathered}
$$

Therefore;

$$
\begin{gathered}
E\left(s_{i}^{2}\right)=\frac{N}{n} \sum_{i=1}^{N} M_{i}\left(M_{i}-m_{i}\right) \frac{\sigma_{i}^{2}}{m_{i}}-\frac{1}{N} \sum_{i=1}^{N} M_{i}\left(M_{i}-\right. \\
\text { miol } 2 m i
\end{gathered}
$$

Also;

$$
\begin{gathered}
E\left(s_{i j}^{2}\right)=E\left\{E\left[E\left(s_{i j}^{2} \mid s_{1}, s_{2}\right)\right]\right\} \\
=E\left\{E\left[\frac{K_{i j}^{2}}{k_{i j}^{2}} \sum_{l=1}^{k_{i j}} E\left(y_{i j l} \mid s_{1}\right)\right]\right\} \\
=E\left\{\frac{M_{i}^{2}}{m_{i}^{2}} E\left[\sum_{j=1}^{m_{i}} \frac{K_{i j}^{2}}{k_{i j}^{2}} \sum_{l=1}^{k_{i j}} E\left(y_{i j l} \mid s_{1}\right)\right]\right\} \\
=E\left\{\frac{M_{i}^{2}}{m_{i}^{2}} E\left[\sum_{j=1}^{M_{i}} \frac{K_{i j}^{2}}{k_{i j}^{2}} \sigma_{i j}^{2}\right]\right\} \\
=E\left\{\sum_{i=1}^{N} \frac{M_{i}^{2}}{m_{i}^{2}} E\left[\sum_{j=1}^{M_{i}} \frac{K_{i j}^{2}}{k_{i j}^{2}} \sigma_{i j}^{2}\right]\right\} \\
=E\left\{\sum_{i=1}^{N} z_{i} \frac{M_{i}^{2}}{m_{i}^{2}} \sum_{j=1}^{M_{i}} K_{i j}\left(K_{i j}-k_{i j}\right) \frac{\sigma_{i j}^{2}}{k_{i j}}\right\} \\
=\frac{n}{N}\left\{\frac{N^{2}}{n^{2}} \sum_{i=1}^{N} \frac{M_{i}}{m_{i}} \sum_{i=1}^{M_{i}} K_{i j}\left(K_{i j}-k_{i j}\right) \frac{\sigma_{i j}^{2}}{k_{i j}}\right\} \\
=\frac{N}{n} \sum_{i=1}^{N} \frac{M_{i}}{m_{i}} \sum_{i=1}^{M_{i}} K_{i j}\left(K_{i j}-k_{i j}\right) \frac{\sigma_{i j}^{2}}{k_{i j}}
\end{gathered}
$$

Therefore;

$$
E\left(s_{i j}^{2}\right)=\frac{N}{n} \sum_{i=1}^{N} \frac{M_{i}}{m_{i}} \sum_{i=1}^{M_{i}} K_{i j}\left(K_{i j}-k_{i j}\right) \frac{\sigma_{i j}^{2}}{k_{i j}}
$$

Combining equations (30), (31) and (32), we have;

$$
\begin{aligned}
E\left\{\hat{V}\left(\hat{Y}_{3 N P E}\right)\right\}= & N(N-n) \frac{\sigma_{1}^{2}}{n} \\
& +\frac{N}{n} \sum_{i=1}^{N} M_{i}\left(M_{i}-m_{i}\right) \frac{\sigma_{i}^{2}}{m_{i}} \\
& +\frac{N}{n} \sum_{i=1}^{N} \frac{M_{i}}{m_{i}} \sum_{j=1}^{M_{i}} K_{i j}\left(K_{i j}-k_{i j}\right) \frac{\sigma_{i j}^{2}}{k_{i j}} \\
& =V\left(\hat{Y}_{3 N P E}\right)
\end{aligned}
$$

That is;

$$
E\left\{\widehat{V}\left(\widehat{Y}_{3 N P E}\right)\right\}=V\left(\widehat{Y}_{3 N P E}\right)
$$

Hence, $\widehat{V}\left(\widehat{Y}_{3 N P E}\right)$ is an unbiased sample estimator of the proposed estimator $\left(\widehat{Y}_{3 N P E}\right)$ in three-stage cluster sampling design.

This estimator, $\hat{Y}_{3 N P E}$, is then compared with these seven conventional three stage cluster sampling design estimators:

$$
\begin{aligned}
& \text { i. } \widehat{Y}_{H H}=\frac{1}{n} \sum_{i=1}^{n} \frac{\underline{Y}_{i . .}}{P_{i}} \\
& \text { ii. } \hat{Y}_{H H G}=M_{0} \frac{\sum_{j=}^{m_{i}} \sum_{i=1}^{n} \hat{y}_{i j}}{\sum_{i=1}^{n} M_{i}} \\
& \text { iii. } \hat{Y}_{H T}=\frac{1}{n} \sum_{i=1}^{n} \frac{1}{\hat{Y}_{i . .}} \\
& \quad \text { iv. } \widehat{Y}_{R H C}=\sum_{g=1}^{n} \frac{z_{g}}{z_{g}} M_{g} \bar{Y}_{g . .} \\
& \text { v. } \hat{Y}_{C}=\sum_{i=1}^{n} \sum_{j=1}^{m_{i}} \sum_{u=1}^{k_{i j}} y_{i j u} \\
& \text { vi. } \hat{Y}_{T}=\sum_{i=1}^{n} \sum_{j=1}^{m_{i}} \sum_{u=1}^{k_{i j}} y_{i j u}
\end{aligned}
$$

and

$$
\text { vii. } \hat{Y}_{O}=\frac{N}{n} \sum_{i=1}^{n} \frac{M_{i}}{m_{i}} \sum_{j=1}^{m_{i}} \frac{K_{i j}}{k_{i j}} \sum_{u=1}^{k_{i j}} y_{i j u}
$$

\section{Data Used for this Study}

There are eight (8) categories of data sets used in this paper. The first four (4) data sets were obtained fro $\mathrm{m}[1],[2],[3]$ and[4] respectively. The second four (4) data sets used are of secondary type and were collected fro $\mathrm{m}[16]$ and[17] where we constructed a sampling frame from all diabetic patients with chronic eye disease (Glaucoma and Retinopathy) in the twenty-five (25) Local Government Areas of the state between 2005 and 2008.

\section{Results}

The estimates obtained with the aid of software developed using Visual Basic C++ Programming Language[18] are given in tables $1-12$ for the illustrated and the real-life data respectively. 
Table 1. Estimated Population Totals using Illustrated Data

\begin{tabular}{|c|c|c|c|c|}
\hline \multirow{2}{*}{ Estimator } & \multicolumn{4}{|c|}{ Cases } \\
\cline { 2 - 5 } & I & II & III & IV \\
\hline$\hat{Y}_{H H}$ & 400 & 98,983 & 36 & 13,898 \\
\hline$\hat{Y}_{H H G}$ & 440 & 111,310 & 22 & 14,000 \\
\hline$\widehat{Y}_{H T}$ & 479 & 139,527 & 27 & 14,791 \\
\hline$\hat{Y}_{R H C}$ & 437 & 98,830 & 31 & 15,214 \\
\hline$\hat{Y}_{C}$ & 385 & 131,675 & 39 & 13,963 \\
\hline$\hat{Y}_{T}$ & 456 & 102,635 & 34 & 14,576 \\
\hline$\hat{Y}_{O}$ & 397 & 141,194 & 24 & 13,950 \\
\hline$\hat{Y}_{3 N P E}$ & 492 & 99,136 & 42 & 15,016 \\
\hline
\end{tabular}

Table 2. Est imated Population Totals using Real Life Data

\begin{tabular}{|c|c|c|c|c|}
\hline Estimator & POP1 & POP2 & POP3 & POP4 \\
\hline$\widehat{Y}_{H H}$ & 26,022 & 26,541 & 28,428 & 29,356 \\
\hline$\hat{Y}_{H H G}$ & 24,355 & 25,019 & 25,162 & 28,610 \\
\hline$\hat{Y}_{H T}$ & 25,514 & 27,197 & 28,731 & 29,096 \\
\hline$\hat{Y}_{R H C}$ & 26,043 & 26,428 & 27,301 & 27,451 \\
\hline$\hat{Y}_{C}$ & 24,420 & 25,321 & 26,851 & 28,365 \\
\hline$\hat{Y}_{T}$ & 25,804 & 27,197 & 27,609 & 29,472 \\
\hline$\hat{Y}_{O}$ & 27,204 & 28,124 & 28,631 & 29,251 \\
\hline$\hat{Y}_{3 N P E}$ & 26,151 & 26,625 & 27,511 & 28,090 \\
\hline
\end{tabular}

Table 3. Biases of Est imat ed Population Totals using Illustrat ed Data

\begin{tabular}{|c|c|c|c|c|}
\hline Estimator & Cases I & Cases II & Cases III & Cases IV \\
\hline$\widehat{Y}_{H H}$ & 29 & 516 & 2 & 446 \\
\hline$\hat{Y}_{H H G}$ & 19 & 337 & 8 & 540 \\
\hline$\hat{Y}_{H T}$ & 29 & 427 & 3 & 133 \\
\hline$\hat{Y}_{R H C}$ & 15 & 364 & 2 & 461 \\
\hline$\hat{Y}_{C}$ & 25 & 309 & 1 & 265 \\
\hline$\hat{Y}_{T}$ & 33 & 463 & 9 & 369 \\
\hline$\hat{Y}_{O}$ & 13 & 261 & 3 & 476 \\
\hline$\hat{Y}_{3 N P E}$ & 11 & 219 & 1 & 112 \\
\hline
\end{tabular}

Table 4. Biases of Estimated Population Totals using Real Life Data

\begin{tabular}{|c|c|c|c|c|}
\hline Estimator & POP1 & POP2 & POP3 & POP4 \\
\hline$\hat{Y}_{H H}$ & 196 & 136 & 136 & 125 \\
\hline$\hat{Y}_{H H G}$ & 147 & 176 & 127 & 168 \\
\hline$\hat{Y}_{H T}$ & 122 & 117 & 114 & 128 \\
\hline$\hat{Y}_{R H C}$ & 155 & 153 & 134 & 118 \\
\hline$\hat{Y}_{C}$ & 127 & 146 & 151 & 120 \\
\hline$\hat{Y}_{T}$ & 164 & 156 & 124 & 195 \\
\hline$\hat{Y}_{O}$ & 137 & 137 & 137 & 158 \\
\hline$\hat{Y}_{3 N P E}$ & 112 & 104 & 103 & 107 \\
\hline
\end{tabular}

Table 5. Variances of the Est imated Population Totals using Illustrated Data

\begin{tabular}{|c|c|c|c|c|}
\hline $\begin{array}{c}\text { Estimato } \\
\mathbf{r}\end{array}$ & Case I & Case II & Case III & Case IV \\
\hline$\widehat{V}\left(\widehat{Y}_{H H}\right)$ & 584.3718 & $163,955.9954$ & 3.0753 & $2,012.5838$ \\
\hline$\widehat{V}\left(\widehat{Y}_{H H G}\right)$ & 498.0637 & $146,797.7757$ & 2.9402 & $1,958.2391$ \\
\hline$\widehat{V}\left(\widehat{Y}_{H T}\right)$ & 444.7838 & $131,942.6350$ & 1.6489 & $1,861.7476$ \\
\hline$\widehat{V}\left(\widehat{Y}_{R H C}\right)$ & 425.8846 & $109,764.4333$ & 1.6226 & $1,844.8793$ \\
\hline$\widehat{V}\left(\widehat{Y}_{C}\right)$ & 418.4156 & $107,030.5504$ & 1.6201 & $1,842.4048$ \\
\hline$\widehat{V}\left(\hat{Y}_{T}\right)$ & 315.7266 & $96,800.8501$ & 1.5117 & $1,834.4763$ \\
\hline$\widehat{V}\left(\widehat{Y}_{O}\right)$ & 296.8283 & $94,624.7532$ & 1.1674 & $1,810.5586$ \\
\hline$\widehat{V}\left(\hat{Y}_{3 N P E}\right)$ & 274.6806 & $91,963.5764$ & 1.1105 & $1,619.5559$ \\
\hline
\end{tabular}

Table 6. Variances of the Est imated Population Totals using Real Life Data

\begin{tabular}{|c|c|c|c|c|}
\hline Estimator & \multicolumn{2}{|c|}{ POP1 POP2 } & POP3 & POP4 \\
\hline$\hat{V}\left(\hat{Y}_{H H}\right)$ & 17,464 & 16,636 & 15,360 & 15,146 \\
\hline$\widehat{V}\left(\widehat{Y}_{H H G}\right)$ & 15,714 & 16,286 & 14,139 & 15,100 \\
\hline$\hat{V}\left(\hat{Y}_{H T}\right)$ & 13,419 & 14,626 & 11,493 & 13,315 \\
\hline$\widehat{V}\left(\hat{Y}_{R H C}\right)$ & 11,684 & 11,788 & 11,398 & 12,396 \\
\hline$\widehat{V}\left(\hat{Y}_{C}\right)$ & 9,985 & 11,532 & 10,938 & 12,239 \\
\hline$\widehat{V}\left(\hat{Y}_{T}\right)$ & 9,749 & 11,441 & 10,532 & 12,069 \\
\hline$\widehat{V}\left(\hat{Y}_{O}\right)$ & 9,568 & 10,507 & 10,330 & 11,087 \\
\hline$\hat{V}\left(\widehat{Y}_{3 N P E}\right)$ & 9,118 & 9,726 & 9,884 & 10,612 \\
\hline
\end{tabular}

Table 7. Standard Error for Est imated Population Total using Illustrated Data

\begin{tabular}{|c|c|c|c|c|}
\hline Estimator & Case I & Case II & Case III & Case IV \\
\hline$\hat{Y}_{H H}$ & 24.1738 & 404.9148 & 1.7537 & 44.8618 \\
\hline$\hat{Y}_{H H G}$ & 22.3173 & 383.1420 & 1.7147 & 44.2520 \\
\hline$\hat{Y}_{H T}$ & 21.0899 & 363.2391 & 1.2841 & 43.1480 \\
\hline$\hat{Y}_{R H C}$ & 20.6370 & 331.3072 & 1.2738 & 42.9521 \\
\hline$\widehat{Y}_{C}$ & 20.4552 & 327.1552 & 1.2728 & 42.9232 \\
\hline$\hat{Y}_{T}$ & 17.7687 & 311.1283 & 1.2295 & 42.8308 \\
\hline$\hat{Y}_{O}$ & 17.2287 & 307.6114 & 1.0805 & 42.5507 \\
\hline$\hat{Y}_{3 N P E}$ & 16.5785 & 302.2597 & 1.0538 & 40.2437 \\
\hline
\end{tabular}

Table 8. Standard Error for Est imated Population Total using Real Life Data

\begin{tabular}{|c|c|c|c|c|}
\hline Estimator & \multicolumn{1}{|c}{ POP1 } & \multicolumn{1}{c|}{ POP2 } & \multicolumn{1}{c|}{ POP3 } & POP4 \\
\hline$\widehat{Y}_{H H}$ & 132.1502 & 128.9710 & 130.6399 & 123.0702 \\
\hline$\widehat{Y}_{H H G}$ & 125.3573 & 127.6163 & 125.3413 & 122.8823 \\
\hline$\widehat{Y}_{H T}$ & 115.8417 & 120.9390 & 113.0051 & 115.3810 \\
\hline$\widehat{Y}_{R H C}$ & 108.0937 & 108.5724 & 112.5340 & 111.3351 \\
\hline$\widehat{Y}_{C}$ & 99.9287 & 107.3872 & 110.2437 & 110.6305 \\
\hline$\widehat{Y}_{T}$ & 98.7379 & 106.9641 & 108.1752 & 109.8593 \\
\hline$\widehat{Y}_{O}$ & 97.8150 & 102.5056 & 107.1344 & 105.2964 \\
\hline$\widehat{Y}_{3 N P E}$ & 95.4893 & 98.6229 & 104.7944 & 103.0137 \\
\hline
\end{tabular}


Table 9. 95\% Confident Intervals for Estimated Population using Illustrated Data

\begin{tabular}{|c|c|c|c|c|}
\hline Estimator & Case I & Case II & Case III & Case IV \\
\hline$\hat{Y}_{H H}$ & $(353,447)$ & $(98189,99777)$ & $(33,39)$ & $(13810,13986)$ \\
\hline$\hat{Y}_{H H G}$ & $(396,484)$ & $(110559,112061)$ & $(19,25)$ & $(13913,14087)$ \\
\hline$\hat{Y}_{H T}$ & $(438,520)$ & $(138815,140239)$ & $(24,30)$ & $(14706,14876)$ \\
\hline$\hat{Y}_{R H C}$ & $(397,477)$ & $(98181,99479)$ & $(29,33)$ & $(15130,15298)$ \\
\hline$\hat{Y}_{C}$ & $(345,425)$ & $(131034,132316)$ & $(37,41)$ & $(13879,14047)$ \\
\hline$\hat{Y}_{T}$ & $(421,491)$ & $(102025,103245)$ & $(32,36)$ & $(14492,14660)$ \\
\hline$\hat{Y}_{O}$ & $(363,431)$ & $(140591,141797)$ & $(22,26)$ & $(13867,14033)$ \\
\hline$\hat{Y}_{3 N P E}$ & $(460,524)$ & $(98542,99730)$ & $(40,44)$ & $(14939,15095)$ \\
\hline
\end{tabular}

Table 10. $95 \%$ Confident Intervals for Estimated Population Totals using Real Life Data

\begin{tabular}{|c|c|c|c|c|}
\hline Estimator & Population 1 & Population 2 & Population 3 & Population 4 \\
\hline$\hat{Y}_{H H}$ & $(25760,26290)$ & $(26290,26790)$ & $(28170,28680)$ & $(29110,29600)$ \\
\hline$\widehat{Y}_{H H G}$ & $(24110,24600)$ & $(24770,25270)$ & $(24920,25410)$ & $(28370,28850)$ \\
\hline$\widehat{Y}_{H T}$ & $(25290,25740)$ & $(26960,27430)$ & $(28510,28950)$ & $(26870,27320)$ \\
\hline$\widehat{Y}_{R H C}$ & $(25830,26250)$ & $(26220,26640)$ & $(27080,27520)$ & $(27230,27670)$ \\
\hline$\widehat{Y}_{C}$ & $(24220,24620)$ & $(25110,25530)$ & $(26630,27070)$ & $(28150,28580)$ \\
\hline$\hat{Y}_{T}$ & $(25610,26000)$ & $(26990,27410)$ & $(27400,27820)$ & $(29260,29690)$ \\
\hline$\hat{Y}_{O}$ & $(27010,27400)$ & $(27920,28320)$ & $(28420,28840)$ & $(29040,29460)$ \\
\hline$\hat{Y}_{3 N P E}$ & $(25960,26340)$ & $(26410,26800)$ & $(27310,27720)$ & $(27890,28290)$ \\
\hline
\end{tabular}

Table 11. Coefficient of Variation for Est imated Population Totals using Illustrated Data

\begin{tabular}{|c|c|c|c|c|}
\hline Estimator & Case I & Case II & Case III & Case IV \\
\hline$\widehat{Y}_{H H}$ & $6.04 \%$ & $0.41 \%$ & $4.87 \%$ & $0.32 \%$ \\
\hline$\widehat{Y}_{H H G}$ & $5.07 \%$ & $0.34 \%$ & $7.79 \%$ & $0.32 \%$ \\
\hline$\widehat{Y}_{H T}$ & $4.40 \%$ & $0.26 \%$ & $4.76 \%$ & $0.29 \%$ \\
\hline$\widehat{Y}_{R H C}$ & $4.72 \%$ & $0.34 \%$ & $4.11 \%$ & $0.28 \%$ \\
\hline$\widehat{Y}_{C}$ & $5.31 \%$ & $0.25 \%$ & $3.26 \%$ & $0.31 \%$ \\
\hline$\widehat{Y}_{T}$ & $3.90 \%$ & $0.30 \%$ & $3.62 \%$ & $0.29 \%$ \\
\hline$\widehat{Y}_{O}$ & $4.34 \%$ & $0.22 \%$ & $4.50 \%$ & $0.31 \%$ \\
\hline$\widehat{Y}_{3 N P E}$ & $3.98 \%$ & $0.31 \%$ & $2.51 \%$ & $0.27 \%$ \\
\hline
\end{tabular}

Table 12. Coefficient of Variation for Estimated Population Totals using Real Life Data

\begin{tabular}{|c|c|c|c|c|}
\hline \multirow{2}{*}{ Estimator } & \multicolumn{4}{|c|}{ Populations } \\
\cline { 2 - 5 } & $\mathbf{1}$ & $\mathbf{2}$ & $\mathbf{3}$ & $\mathbf{4}$ \\
\hline$\widehat{Y}_{H H}$ & $0.44 \%$ & $0.43 \%$ & $0.42 \%$ & $0.42 \%$ \\
\hline$\hat{Y}_{H H G}$ & $0.51 \%$ & $0.51 \%$ & $0.44 \%$ & $0.43 \%$ \\
\hline$\widehat{Y}_{H T}$ & $0.45 \%$ & $0.44 \%$ & $0.37 \%$ & $0.34 \%$ \\
\hline$\widehat{Y}_{R H C}$ & $0.42 \%$ & $0.41 \%$ & $0.37 \%$ & $0.41 \%$ \\
\hline$\widehat{Y}_{C}$ & $0.37 \%$ & $0.42 \%$ & $0.38 \%$ & $0.33 \%$ \\
\hline$\hat{Y}_{T}$ & $0.38 \%$ & $0.33 \%$ & $0.36 \%$ & $0.37 \%$ \\
\hline$\hat{Y}_{O}$ & $0.36 \%$ & $0.36 \%$ & $0.36 \%$ & $0.36 \%$ \\
\hline$\widehat{Y}_{3 N P E}$ & $0.34 \%$ & $0.33 \%$ & $0.35 \%$ & $0.37 \%$ \\
\hline
\end{tabular}

\section{Discussion of Results}

The estimation methods given in equation (10) was applied to four different illustrated data (Cases I - IV) and four real life data (Populations 1 - 4). The population totals obtained for illustrated data are given in table 1 while the population totals obtained for real life data are given in table 2. Table 3 give the biases of the estimated population totals for illustrated data for our own estimator as $11,219,1$, and 112 for cases I - IV respectively while table 4 gives that of the four life data sets as $112,104,103$, and 107 respectively. This implies that our own estimator has the least biases using both data sets. Table 5 shows the variances obtained using illustrated data for our own estimator as 274.6806, 91963.5764, 1.1105 and 1619.5559 for cases I - IV respectively while table 6 shows that of life data sets as 9118.2037, 9726.4809, 9883.6215 and 10611.8216 respectively meaning that our own estimator has the least variances using both data sets. Table 7 shows the obtained standard errors for the estimated population totals using illustrated data for our own estimator as $16.5785,302.2597$, 1.0538 and 40.2437 for cases I - IV respectively while table 8 shows that of life data sets as $95.4893,98.6229,104.7944$ and 103.0137 respectively meaning that our own estimator have the least standard errors using both data sets.

The confidence intervals of the estimated populations in table 1 are given in table 9 for $\alpha=, 5 \%$. The confidence intervals of the estimated populations in table 2 are given in 
table 10 for $\alpha=5 \%$ which shows that all the estimated population totals fall within the computed intervals as expected. For our own estimator, table 11 gives the coefficients of variations for the estimated population totals using illustrated data as $3.98 \%, 0.31 \%, 2.51 \%$ and $0.27 \%$ for cases I - IV respectively while table 12 gives that of life data sets as $0.34 \%, 0.33 \%, 0.35 \%$ and $0.37 \%$ respectively which means that our newly proposed three stage cluster estimator has the least coefficient of variation, hence it is preferred.

\section{Conclusions}

The alternative estimation method of population allows the use of certain number of visits to the venues (hospitals) within the clusters (cities) and a more precise (minimum mean square error) estimate was obtained and the estimates presented indicate that substantial reduction in the variances was obtained through the use of newly proposed estimator. We also observed that irrespective of the data considered, the variance of newly proposed estimator is always less than those of already existing estimators in three-stage cluster sampling designs. The newly proposed estimator $\left(\widehat{Y}_{3 N P E}\right)$ is preferred to the already existing estimators considered in this study and is therefore recommended.

\section{REFERENCES}

[1] Horvitz, D. G. and D. J. Thompson, "A Generalization of Sampling without Replacement from a Finite Universe", Journal of American Statistical Association, vol. 47 663-685, 1952.

[2] Raj, D., The Design of Sample Survey, McGraw-Hill, Inc., New York, USA, 1972.

[3] Cochran, W.G., Sampling Techniques, Third Edition, John Wiley and Sons, New York, 1977.

[4] Okafor, F., Sample Survey Theory with Applications, Afro-Orbis Publications, Nigeria, 2002.
[5] Kish, L., Survey Sampling, John Wiley and Sons, New York, 1967.

[6] Adams, J. L., S. L. Wickstrom, M. J. Burgess, P. P. Lee, and J. J. Escarce, "Sampling Patients within Phy sician Practices and Health Plans: Multistage Cluster Samples in Health Services Research", The Global Journal for Improving Health Care Delivery and Policy, vol. 38: 1625-1640, 2003.

[7] Kuk, A., "Estimation of Distribution Functions and Medians under Samp ling with Unequal Probabilities", Biometrika, vol. 75, 97-103, 1988.

[8] Goldstein, H., Multilevel Statistical Models, Halstead Press, New York, 1995.

[9] Tate, J.E. and M.G. Hudgens, "Estimating Population Size with Two-stage and Three-stage sampling designs", American Journal of Epidemiology,vol. 165 no.11, 1314-1320, 2007.

[10] Kalton, G., Introduction to Survey Sampling, Sage Publications, Thousand Oaks, C.A., 1983.

[11] Henry, G. T., Practical Sampling Thousand Oaks, C.A.: Sage Publications, 1990.

[12] Fink, A., How To Sample In Survey, C.A. Sage Publications, Thousand Oaks, 2002.

[13] Kendall, M. G. and A. S. Stuart, The Advanced Theory of Statistics, Hafner Publishing Company, New York, 1968.

[14] Thompson, S. K., Sampling John Wiley and Sons, New York, 1992.

[15] Nafiu, L. A., An Alternate Estimation Method for Multistage Cluster Sampling in Finite Population, Unpublished Ph.D Thesis, University of Ilorin, Ilorin, Nigeria, 2012.

[16] Niger State, Niger State Statistical Year Book, Niger Press Printing \& Publishing, Nigeria, 2009.

[17] National Bureau of Statistics, Directory of Health Establishments in Nigeria, Abuja Printing Press, Nigeria, 2007.

[18] Hubbard, J. R., Programming with C++, Second Edition. Schaum's Outlines, Tata MoGraw-Hill Publishing Company Limited, New Delhi, 2000. 